\title{
Slow magnetoacoustic waves in coronal loops: EIT and TRACE
}

\author{
E. Robbrecht ${ }^{1,2}$, E. Verwichte ${ }^{2}$, D. Berghmans ${ }^{2}$, J. F. Hochedez ${ }^{2}$, S. Poedts ${ }^{1, \star}$, and V. M. Nakariakov ${ }^{3}$ \\ 1 Centre for Plasma Astrophysics, K.U. Leuven, Celestijnenlaan 200B, 3001 Leuven, Belgium \\ 2 Royal Observatory of Belgium, Ringlaan 3, 1180 Brussel, Belgium \\ 3 Physics Department, University of Warwick, Coventry CV4 7AL, UK
}

Received 20 November 2000 / Accepted 6 February 2001

\begin{abstract}
On May 13, 1998 the EIT (Extreme ultraviolet Imaging Telescope) on board of SoHO (Solar and Heliospheric Observatory) and TRACE (Transition Region And Coronal Explorer) instruments produced simultaneous high cadence image sequences of the same active region (AR 8218). TRACE achieved a $25 \mathrm{~s}$ cadence in the Fe IX (171 ̊) bandpass while EIT achieved a 15 s cadence (operating in "shutterless mode", SoHO JOP 80) in the Fe XII (195 $\AA$ ) bandpass. These high cadence observations in two complementary wavelengths have revealed the existence of weak transient disturbances in an extended coronal loop system. These propagating disturbances (PDs) seem to be a common phenomenon in this part of the active region. The disturbances originate from small scale brightenings at the footpoints of the loops and propagate along the loops. The projected propagation speeds roughly vary between 65 and $150 \mathrm{~km} \mathrm{~s}^{-1}$ for both instruments which is close to and below the expected sound speed in the coronal loops. The measured slow magnetoacoustic propagation speeds seem to suggest that the transients are sound (or slow) wave disturbances. This work differs from previous studies in the sense that it is based on a multi-wavelength observation of an entire loop bundle at high cadence by two EUV imagers. The observation of sound waves along the same path shows that they propagate along the same loop, suggesting that loops contain sharp temperature gradients and consist of either concentric shells or thin loop threads, at different temperatures.
\end{abstract}

Key words. Sun: activity - Sun: corona - Sun: oscillations - Sun: UV radiation - MHD - waves

\section{Introduction}

In this paper we report on the detection and study of propagating disturbances (PDs) along coronal loops.

Our study is focused on active region AR8218, using image sequences of JOP80 (Clette et al. 1998). This JOP (Joint Operation Project) was performed on May 13, 1998 and produced simultaneously high cadence image sequences of the same active region in different wavelengths. The analysis in this paper is based on observations of two instruments measuring in two different wavelengths: EIT (Extreme ultraviolet Imaging Telescope) on board SoHO (Solar and Heliospherical Observatory) measures in $195 \AA$ and TRACE (Transition Region And Coronal Explorer) measures in $171 \AA$. A previous study on the EIT data has been done by Robbrecht et al. (1999) in which they discuss the detection of PDs in coronal loops. They show that slow magnetoacoustic waves are a possible interpretation of these PDs. In the present paper we find stronger evidence for this hypothesis, based on a study of the TRACE

Send offprint requests to: E. Verwichte, e-mail: Erwin.VERWICHTE@oma.be

* Research Associate of the Fund for Scientific Research in Flanders (FWO-Vlaanderen). data (part of JOP80) and the comparison between the TRACE and the EIT data.

There exist many observations of time variability in active region loops. Aschwanden et al. (1999) give a very broad overview of spatial oscillations of coronal loops and coronal loop dynamics in general. Svestka et al. (1982a, 1982b) observed quasi-periodic brightness variations. Roberts et al. (1984) suggested that these quasiperiodic variations might be caused by free, slow-mode MHD (Magneto-HydroDynamic) oscillations of large scale loops. Later they observed phenomena they called "flaring arches": propagating brightenings seen in X-rays and streaming material in $\mathrm{H} \alpha$ in a giant arch preceded by a flare (Martin \& Svestka 1988; Svestka et al. 1989). Harrison (1987) observed for the first time soft X-ray pulsations from the non-flaring Sun. The pulsations originate from a compact active region which lies at one footpoint of a large coronal loop. The periodicity is thought to be produced by a standing wave or a traveling wave "packet" which exists within the loops. With the recent operation of more powerful instruments, the list of observational evidence for variability in coronal loops is largely extended. Brekke et al. (1997) reported of the first observations of high-velocity flows in active region loop systems observed with the CDS (Coronal Diagnostic Spectrometer) 
on SoHO. The velocities observed at a temperature of $240000 \mathrm{~K}$ are of the order of $50-60 \mathrm{~km} \mathrm{~s}^{-1}$. They find, within the same loop system, velocities in opposite directions seen in neighbouring loops. They argue these disturbances are not caused by mass-flows along the loops because that would require velocity values considerably larger than the measured values. Kjeldseth-Moe \& Brekke (1998) investigated loops at temperatures ranging from $10^{4} \mathrm{~K}$ to $2.710^{6} \mathrm{~K}$ using CDS. By investigating Doppler line shifts, velocities from $20 \mathrm{~km} \mathrm{~s}^{-1}$ up to $300 \mathrm{~km} \mathrm{~s}^{-1}$ have been found. They mention magnetoacoustic waves as a possible explanation of the line shifts. Berghmans \& Clette (1999) first discussed a new class of weaker footpoint brightenings that produce wave-like disturbances propagating along quasi-open field lines, which have been further analyzed by Robbrecht et al. (1999) and which will also be studied in this report. Also more recently De Moortel et al. (2000) reported on similar propagating oscillations in large diffuse coronal loops, using TRACE data. Not only longitudinal oscillations, but also transverse oscillations of active region loops have been observed directly (e.g. Aschwanden et al. 1999; Nakariakov et al. 1999).

The phenomenon of propagating disturbances along loops is comparable to moving features observed in polar plumes. DeForest \& Gurman (1998) reported on the observation of outward propagating features in plumes, traveling outward at speeds of $75-150 \mathrm{~km} \mathrm{~s}^{-1}$ along the plume structure. They argued that these moving features are slow magnetoacoustic waves because

1. the propagation speed is always less than the sound speed of the observation;

2. the moving features repeat in quasi-periodic trains; and

3. Doppler shifts have not been reported in plumes for these speeds.

Ofman et al. (1999) reported on an increase in relative intensity of the features with radial distance and found a good match between the observations and a onedimensional analytical model of spherical slow magnetoacoustic waves in a stratified atmosphere.

Nightingale et al. (1999) have analyzed the time variability of EUV brightenings in coronal loops, based on TRACE data in the $171 \AA$ line. They conclude that these small EUV (Extreme UltraViolet) brightenings cannot be associated with upflows of heated plasma by chromospheric evaporation, consequently they also do not contribute to coronal heating. Instead, their near-isothermal density enhancements seem to be caused by compressional waves, which start near the loop footpoints and propagate along the loops with approximate sound speed.

Contrary to the acoustic wave interpretation, Reale et al. (2000) interpreted the evolution of a transient brightening of a coronal loop, observed by TRACE, in terms of an initially empty and cool magnetic flux tube along which plasma motion takes place. This interpretation is inspired by a transient heating episode occurring along the loop. However, Nakariakov et al. (2000) have shown that the propagating disturbances in coronal loops can be interpreted as slow magnetoacoustic waves analogously to waves in coronal holes.

The studies mentioned above are all important in the process of understanding the heating and dynamics of coronal loops. However, up to now an important factor was missing: the ability of comparing observations in different wavelengths. This is a powerful tool since it can be used, not only to observe coronal features instantaneously at different temperatures, but also to check measured values (propagation speeds, inclination angles of loops with respect to the field of view and their interpretation. The importance of this paper lies in this crucial factor of simultaneously observing the same coronal feature at different wavelengths.

In Sect. 2 the data sets and the morphology of the active region are described. Section 3 contains an explanation of the analysis, leading to the slow magnetoacoustic wave interpretation. The results are described in Sect. 4 which are followed by a discussion.

\section{Observations}

On May 13, 1998, the EIT instrument on board SoHO and TRACE telescope have produced a unique image sequence in the context of the multi-instrument campaign SoHO JOP80 (Clette et al. 1998). JOP80 is dedicated to the high-time resolution imaging study of coronal and transition region dynamics (EIT shutterless-mode campaign). The initial aim was to study bright structures (active region loops, bright points) with the highest possible time resolution and wide spatial coverage. First results of this JOP80 have been reported by Berghmans \& Clette (1999); Robbrecht et al. (1999). In JOP80, EIT is the lead instrument, followed by several space-born instruments: SXT (Soft X-ray Telescope), TRACE, MDI (Michelson Doppler Imager), CDS (Coronal Diagnostics Spectrometer), SUMER (Solar Ultraviolet Measurements of Emitted Radiation), as well as two ground based observatories (in La Palma and Sac Peak). The combination of these instruments allows for good statistics of many local events.

\subsection{EIT data}

EIT (Extreme ultraviolet Imaging Telescope, Delaboudinière et al. 1995; Tarbell 1994) achieved an exceptional $15 \mathrm{~s}$ cadence in the FexII bandpass (195 ^) by leaving the instrument's shutter open for 1 hour and operating the CCD in frame transfer mode. EIT collected during the 1 hour JOP 80 run in total 229 images of $128 \times 96$ pixels $(332 \times 249$ arcsec $)$ starting at 17:31:50 UT and ending at 18:29:07 UT on 1998 May 13 , yielding a time interval of $3437 \mathrm{~s}$. Flat-field and grid pattern corrections have been applied to the images. In the EIT data, cosmic rays were identified as exceptional deviations (above the $5 \sigma$ level) in the pixel's light curves 
Table 1. Characteristics of the JOP80 dataset produced by the EIT and TRACE instruments. The bandpasses of EIT Fe XII (195 A) and TRACE Fe IX (171 A) are narrow with a peak formation temperature of respectively 1.6 MK and $1 \mathrm{MK}$. The end time of the TRACE sequence is determined by cosmic rays which damaged the images too much

\begin{tabular}{|c|c|c|}
\hline Instrument & EIT & TRACE \\
\hline Bandpass & $195 \AA$ & $171 \AA$ \\
Ion & Fe XII & Fe IX \\
Level & Corona & Low Corona \\
Temp & $1.6 \mathrm{MK}$ & $1 \mathrm{MK}$ \\
Cadence & $15 \mathrm{~s}$ & $25 \mathrm{~s}$ \\
Pixelsize & $2.59 \operatorname{arcsec} / \mathrm{pix}$ & $0.5 \mathrm{arcsec} / \mathrm{pix}$ \\
starttime & $17 \mathrm{~h} 31 \mathrm{~m} 50 \mathrm{~s}$ & $17 \mathrm{~h} 03 \mathrm{~m} 28 \mathrm{~s}$ \\
endtime & $18 \mathrm{~h} 29 \mathrm{~m} 07 \mathrm{~s}$ & $18 \mathrm{~h} 07 \mathrm{~m} 17 \mathrm{~s}$ \\
\hline
\end{tabular}

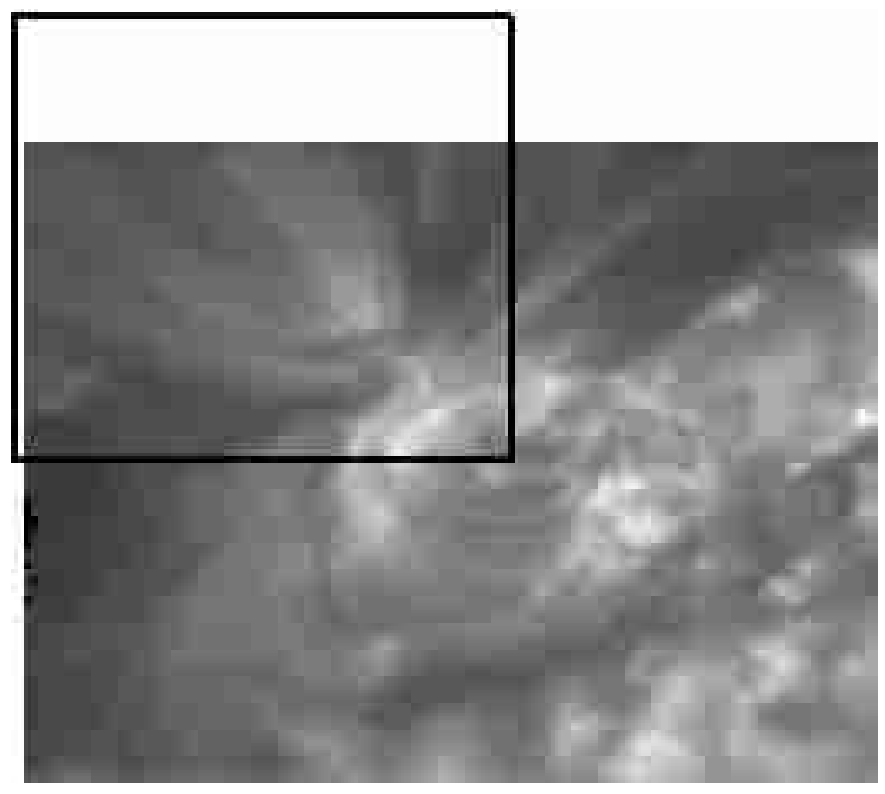

Fig. 1. The field of view of EIT, specified in the full disc in Fig. 3. Superimposed we have drawn the field of view of the TRACE image which is shown in Fig. 2

that appear in one image only. Their values were restored by replacing the pixel's intensity with a linear interpolation from neighbouring images. See Delaboudinière et al. (1995) and Berghmans \& Clette (1999) for more technical details. Due to the solar rotation, the active region shifts to the right of the field of view during the 1 hour observation campaign by less than 4 EIT pixels. This shift was compensated to a subpixel level by determining the maximum of cross-correlation between the images.

\subsection{TRACE data}

The TRACE (Transition Region And Coronal Explorer, Handy et al. 1999) dataset consists of a datacube with a spatial size of $1024 \times 1024$ pixels and a temporal sequence of 147 images at a cadence of $26 \mathrm{~s}$ in the Fe IX bandpass $(171 \AA)$. The image sequence starts at 17:03:28 UT and

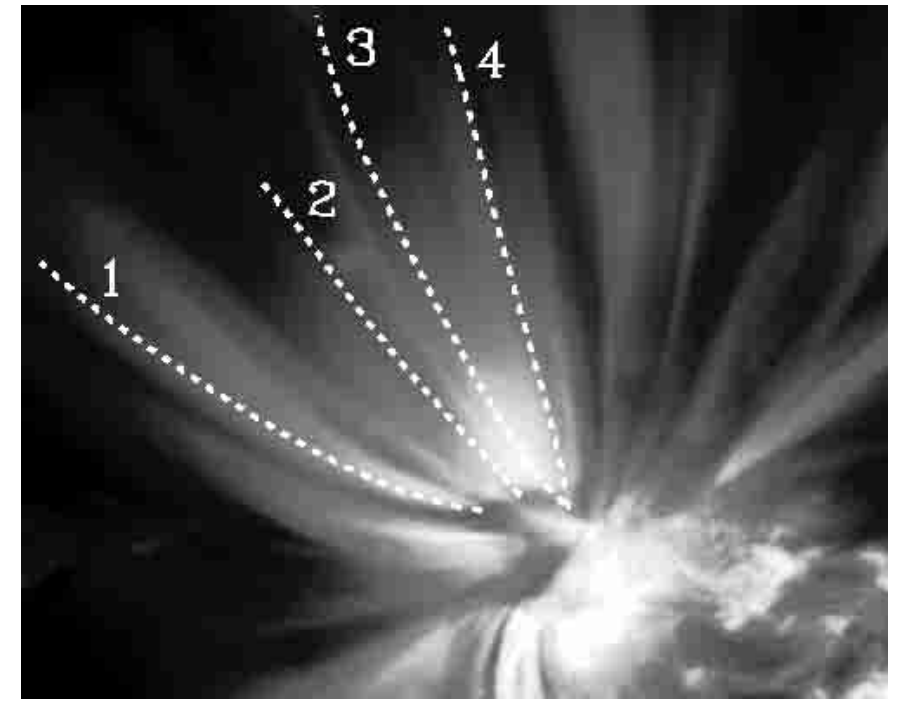

Fig. 2. The field of view of TRACE, specified in Fig. 1. In Figs. 5 to 8 we give the characteristics of the 4 loops tracked here

ends at 18:07:17 UT on 1998 May 13, with a total duration of 3829 s. A subregion of $400 \times 355$ pixels $(200 \times$ 177.5 arcsec) has been selected from the whole field of view. There is not a complete match (spatially and temporally) between the EIT and the TRACE datasets (see Fig. 1 and Table 1 resp. for the spatial and temporal alignment). Due to cosmic rays, the last part of the TRACE sequence cannot be used and therefore there is only half an hour overlap between the two data sets. To clean the TRACE data we used standard procedures found in the SolarSoft TRACE analysis guide (?). The TRACE images suffer greatly from radiation noise spikes due to the radiation belt passages. In the time period between $17 \mathrm{~h} 28$ and $17 \mathrm{~h} 35$ the cosmics are severe. The pixels hit by cosmic rays (exceeding the $6 \sigma$ level) are replaced by a nearby pixel value. We removed the background diffraction pattern by applying a Fourier analysis on the data. The TRACE images were corrected for solar rotation and spacecraft jitter by determining the shift for maximum cross-correlation between the images. The internally co-aligned EIT and TRACE data cubes were then mutually co-aligned on the basis of the image headers pointing information. An improvement on this was achieved by visually identifying a relative large number $(>10)$ of distinctive features that could be recognized in both images. An optimal coordinate transformation is then constructed (least mean square basis) that maps the TRACE image to the EIT image and vice versa. All this results in an EIT-TRACE co-alignment better than 1-2 EIT pixels which is sufficient for the present study.

\subsection{Active region 8218}

The target of the campaign was a relatively small but highly dynamical active region (AR 8218). In a first inventory of the dynamics of this active region, a wide 

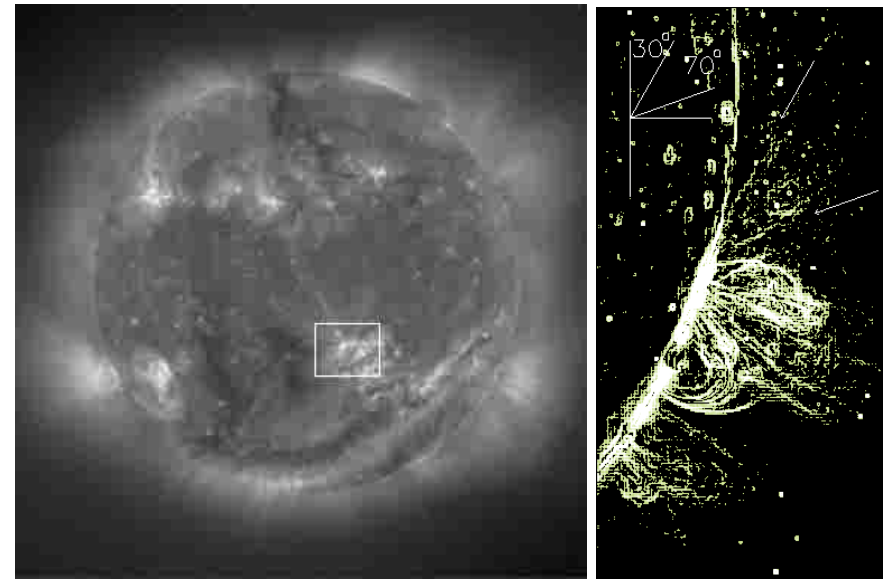

Fig. 3. At the left, an EIT full disc image in the Fe XII (195 $\AA)$ bandpass, taken at $18 \mathrm{~h} 34 \mathrm{~m}$ UT. The rectangle indicates the active region AR 8218, it corresponds to the EIT field of view. At the right, a side view on $\mathrm{AR} 8218$ when crossing the western limb. This image has been processed to enhance the edges of the loop structure

variety of transients was discovered, ranging from a B3.5 flare producing a large plasma flow along pre-existing loops, to EUV versions of active region transient brightenings as previously observed by SXT on board YOHKOH (Berghmans \& Clette 1999; Shimojo 1998). In Fig. 3 (left), we show the overall structure of this active region and focus (Fig. 1) on the bundle of long, magnetic flux tubes that emanates from it to the NE. The width of the EIT field of view is about $2.310^{8} \mathrm{~m}\left(0.34 R_{\odot}\right)$. Taking profit of the solar rotation and assuming that the overall structure of this bundle remained unchanged over a period of a week, we obtained a side view of the active region when it crossed the western limb (Fig. 3, right). From this we estimate that the magnetic flux tubes in the bundle make an angle $\alpha$ with the plane of the sky that ranges from roughly $30^{\circ}$ up to $70^{\circ}$.

\section{Investigating the loops}

Here we focus on an additional, new type of transients that was discovered propagating along the bundle of widely opening magnetic field lines in the NE of the active region. In this region, loops are quite clear. To illustrate the variability of the analyzed loop system we show in Fig. 4 a sequence of 4 running difference images at $171 \AA$, and $195 \AA$. Dark and bright features, outlining the whole length of a loop, suggest that the loops also experience transverse motions. The pattern of diagonal lines in the TRACE sequence of difference images is an instrumental artifact. They are remains of the background diffraction pattern, which are enhanced by the differencing. In a difference movie, we see a continuous outflow of bright disturbances. After a sudden brightening at the footpoint of the fan of loops we detect a quasi-periodical radial outflow of disturbances, along the loops. Figure 4 illustrates the propagation of a PD by making running difference images. The PDs are indicated with a white arrow. Note, that the

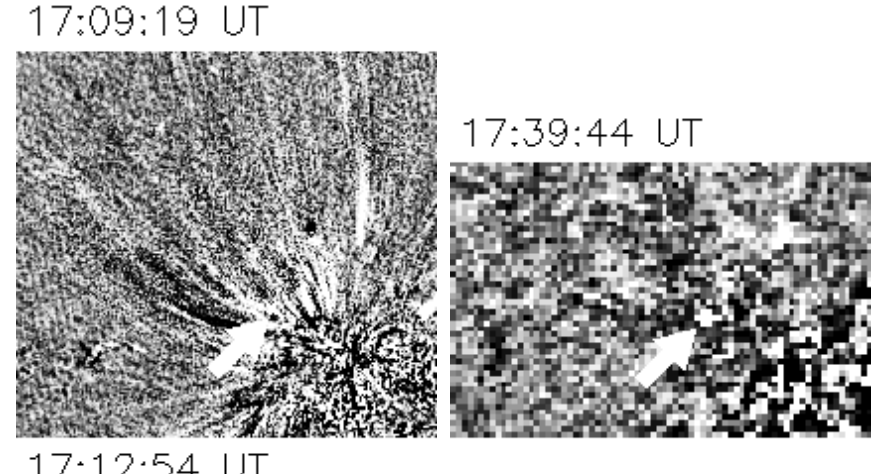

$17: 12: 54$ UT

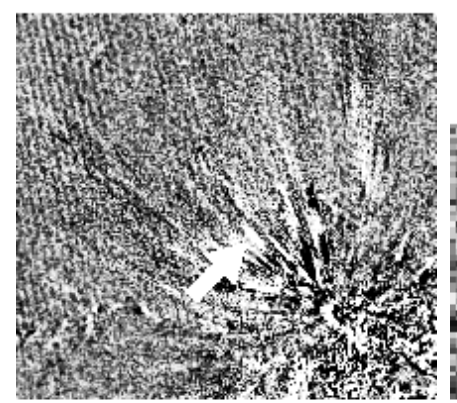

17:41:39 UT

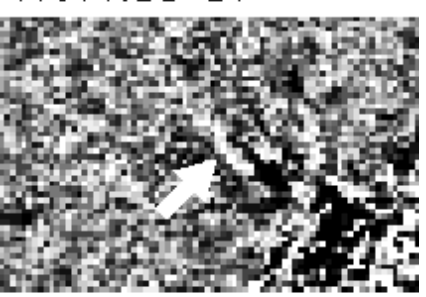

$17: 16: 30$ UT
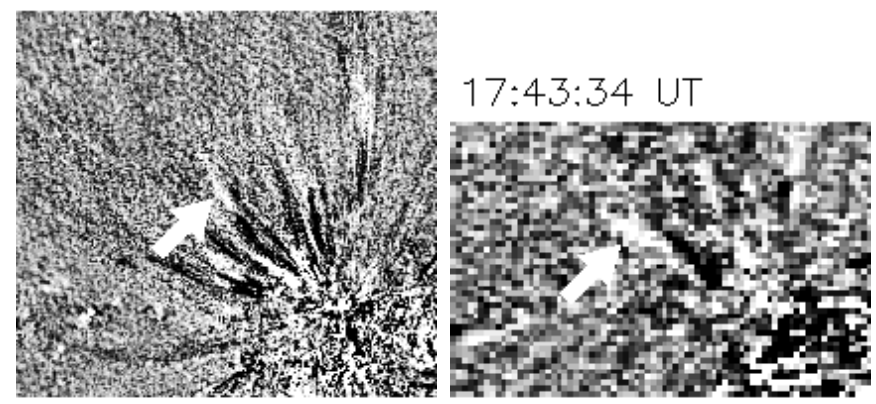

$17: 20: 06$ UT

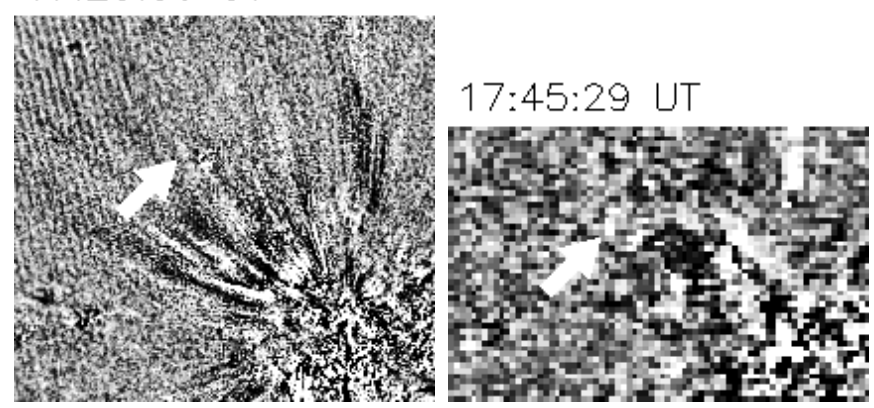

Fig. 4. Two sequences of 4 "running time difference" images are shown, extracted from TRACE $171 \AA$, images (at the left) and from EIT $195 \AA$, images (at the right). The difference images are created by subtracting an image taken $2 \mathrm{~m} 15 \mathrm{~s}$ (for TRACE) and $1 \mathrm{~m} 54 \mathrm{~s}$ (for EIT) earlier than the time mentioned in the upper left corner. We notice that there is no correspondence between the time of TRACE and EIT. White arrows indicate the propagation of a disturbance

sequences of TRACE and EIT do not correspond in time here.

We identified oscillations in several loops during the 1998 May 13 time sequence. Four paths are selected visually for detailed analysis (Fig. 2). We tracked the loops on the TRACE image, which has the higher resolution of 
the two instruments. We then applied a transformation to obtain the same path in the EIT data. The transformation method is described in Sect. 2.2. The width of the path is equal to the pixelsize of the instrument. The path in the EIT data is generally shorter, since the field of view is smaller at the North of the active region, where the loops are tracked. By assuming a semi-circular loop, we visually estimate the length along an entire loop to be $800 \mathrm{Mm}$. The path length (projection onto the field of view) is estimated to be of the order of $100 \mathrm{Mm}$.

\subsection{Slow magnetoacoustic wave interpretation}

In Figs. 5 to 8 we show the temporal evolution (horizontal axis) along the four selected paths (vertical axis). A quadratic fit to each pixel's light curve was calculated. This quadratic fit is taken as the slowly varying, EUV emission background. The relative variations with respect to this background is shown. In this diagram we see propagating disturbances as bright and dark diagonal ridges. From this diagram, the apparent propagation speed $v_{\mathrm{p}}$ of each disturbance along the loop is deduced, by measuring the slope of its respective ridge. This is done by hand. The speed we measure is apparent because we only measure the component projected onto the plane of the sky. Here the interpretation of slow magnetoacoustic waves makes its way. EIT (1.6 MK) measures at a higher temperature than TRACE $(1 \mathrm{MK})$. The sound speed $C_{\mathrm{S}}$ can be expressed in function of temperature alone (Priest 1984). This allows us to derive a formal sound speed:

$$
\begin{aligned}
C_{\mathrm{S}} & =152 \mathrm{~T}^{1 / 2} \mathrm{~m} \mathrm{~s}^{-1}(\mathrm{~T} \text { in } \mathrm{K}), \\
& =152 \mathrm{~km} \mathrm{~s}^{-1} \text { for TRACE data }(171 \AA), \\
& =192 \mathrm{~km} \mathrm{~s}^{-1} \text { for EIT data }(195 \AA) .
\end{aligned}
$$

The expected sound speed is higher for the EIT observations than for the TRACE observations (see Table 1). The apparent propagation speeds $v_{\mathrm{p}}$ which we list in the four tables, are of the order of, but never exceed this derived sound speed. This suggests that these PDs are sonic perturbations.

If we assume this, then the difference between the measured speed and the sound speed is explained by the projection onto the plane of the sky. This means that we can derive from the difference between the apparent speed and the sound speed, the projection angle $\alpha_{\exp }$ between the loop direction and the plane of the sky:

$$
\alpha_{\exp }=\arccos \left(\frac{v_{\mathrm{p}}}{C_{\mathrm{S}}}\right) \text {. }
$$

\subsection{Discussion of measurements}

For each of the four paths we have plotted the time-space diagram for TRACE and EIT, in such a way that the times correspond vertically (Figs. 5 to 8 ). The letters under the diagrams indicate the starting points of the PDs of which we measured the apparent speed. These letters are written in the tables in the column labeled "l". In some cases, a particular disturbance occurs in the two data sets, such that comparison between the two data sets is possible. The tables describe the measured speeds quite in detail. $v_{\mathrm{p}}$ is the apparent speed. We measured this speed for each ridge at the bottom of the path $(<20 \mathrm{Mm}$ starting from the footpoint of the loop which is not the same as the starting point of the path), at the top $(>20 \mathrm{Mm})$ and over the full length of the path. The error is calculated as s.e. $=\sqrt{\sigma^{2} n}$ where $\sigma^{2}$ is the variance and $n$ the number of measurements of the slope of the PD in the diagram. With each speed, there is a corresponding projection angle $\alpha_{\exp }$. This is calculated through formula (3). In the tables we list the interval $\left[\alpha_{\exp }\left(v_{\mathrm{p}}-\right.\right.$ s.e. $), \alpha_{\exp }\left(v_{\mathrm{p}}+\right.$ s.e. $\left.)\right]$. If this interval lies entirely in the interval $\left[30^{\circ}, 70^{\circ}\right]$, for both datasets, then our measurements do not speak against the slow magnetoacoustic wave interpretation.

We now briefly describe the four paths in detail. To simplify the description, we will use the following notation: "Tb" means PD b in the TRACE dataset, "Ea" is PD a in the EIT dataset.

\subsubsection{Path 1}

The footpoint of the loop lies at roughly $10 \mathrm{Mm}$ above the starting point of path 1 . In each diagram we can distinct a broad bright ridges, Tc and Ec. They have an apparent speed of respectively 105 and $124 \mathrm{~km} \mathrm{~s}^{-1}$. The projection angles lie in the ranges $\left[40^{\circ}, 52^{\circ}\right]$ and $\left[45^{\circ}, 54^{\circ}\right]$ resp. Both intervals are acceptable (i.e. lie between $30^{\circ}$ and $\left.70^{\circ}\right)$. Moreover, there is a broad overlap between the two intervals $\left(\left[45^{\circ}, 52^{\circ}\right]\right)$, which indicates that we can be sure to measure PDs in the same loop. Assuming that Tc and Ec have the same physical origin, we could measure a period of 30 min between these two PDs. Furthermore there are thinner PDs with roughly the same speed as in Tc and Ec, apart from PD Td, which propagates at a remarkably lower speed. Nevertheless, the angle deduced for PD Td is still acceptable.

\subsubsection{Path 2}

The footpoint of the loop lies at roughly $10 \mathrm{Mm}$ above the starting point of path 2. Remark that the EIT frame was to small to contain the whole path. An overall clear diagonal structure is visible in both diagrams. The average (weighted) propagation speed in the TRACE frame is $95 \pm$ $1 \mathrm{~km} \mathrm{~s}^{-1}$ inferring an expected angle of $\alpha_{\exp }=51 \pm 1^{\circ}$ and $111 \pm 3 \mathrm{~km} \mathrm{~s}^{-1}$ in the EIT frame, inferring an expected angle of $\alpha_{\exp }=55 \pm 1^{\circ}$. In calculating the average speed PD Ta was not taken into account, since the footpoint lies before the TRACE time window. PD Tb seems broad and diffuse, making speed determination difficult and also prone to systematic error. The large s.e. errors hint to that. PD Tg shows a notable turn from slow to faster speeds, so its total speed measure is underestimated with 


\begin{tabular}{|c|c|c|c|c|c|c|c|c|c|}
\hline \multicolumn{5}{|c|}{ TRACE } & \multicolumn{5}{|c|}{ EIT } \\
\hline l & $v_{\mathrm{p}}(<20 \mathrm{Mm})$ & $v_{\mathrm{p}}(>20 \mathrm{Mm})$ & $v_{\mathrm{p}}$ & \multirow[t]{2}{*}{$\alpha_{\exp }$} & l & $v_{\mathrm{p}}(<20 \mathrm{Mm})$ & $v_{\mathrm{p}}(>20 \mathrm{Mm})$ & $v_{\mathrm{p}}$ & \multirow[t]{2}{*}{$\alpha_{\exp }$} \\
\hline & \multicolumn{3}{|c|}{$\left(\right.$ in $\left.\mathrm{km} \mathrm{s}^{-1}\right)$} & & & \multicolumn{3}{|c|}{$\left(\right.$ in $\left.\mathrm{km} \mathrm{s}^{-1}\right)$} & \\
\hline $\bar{A}$ & $71 \pm 12$ & $121 \pm 13$ & $88 \pm 8$ & $50^{\circ}-58^{\circ}$ & - & - & - & - & \\
\hline B & $74 \pm 6$ & $100 \pm 25$ & $88 \pm 5$ & $52^{\circ}-57^{\circ}$ & - & - & - & - & \\
\hline $\mathrm{C}$ & $114 \pm 8$ & & $105 \pm 11$ & $40^{\circ}-52^{\circ}$ & - & - & - & - & \\
\hline $\mathrm{D}$ & $64 \pm 11$ & & $75 \pm 21$ & $50^{\circ}-70^{\circ}$ & A & $125 \pm 8$ & $121 \pm 21$ & $134 \pm 11$ & $41^{\circ}-50^{\circ}$ \\
\hline $\mathrm{E}$ & $74 \pm 11$ & $119 \pm 23$ & $94 \pm 12$ & $46^{\circ}-57^{\circ}$ & - & - & - & - & \\
\hline $\mathrm{F}$ & $104 \pm 20$ & $126 \pm 27$ & $119 \pm 11$ & $31^{\circ}-45^{\circ}$ & - & - & - & - & \\
\hline & - & - & - & - & B & $129 \pm 29$ & $128 \pm 24$ & $126 \pm 14$ & $43^{\circ}-54^{\circ}$ \\
\hline & - & - & - & - & $\mathrm{C}$ & $151 \pm 23$ & $118 \pm 16$ & $124 \pm 12$ & $45^{\circ}-54^{\circ}$ \\
\hline & - & - & - & - & D & $113 \pm 24$ & & $126 \pm 13$ & $43^{\circ}-54^{\circ}$ \\
\hline & - & - & - & - & $\mathrm{E}$ & $116 \pm 10$ & & $124 \pm 10$ & $45^{\circ}-54^{\circ}$ \\
\hline
\end{tabular}

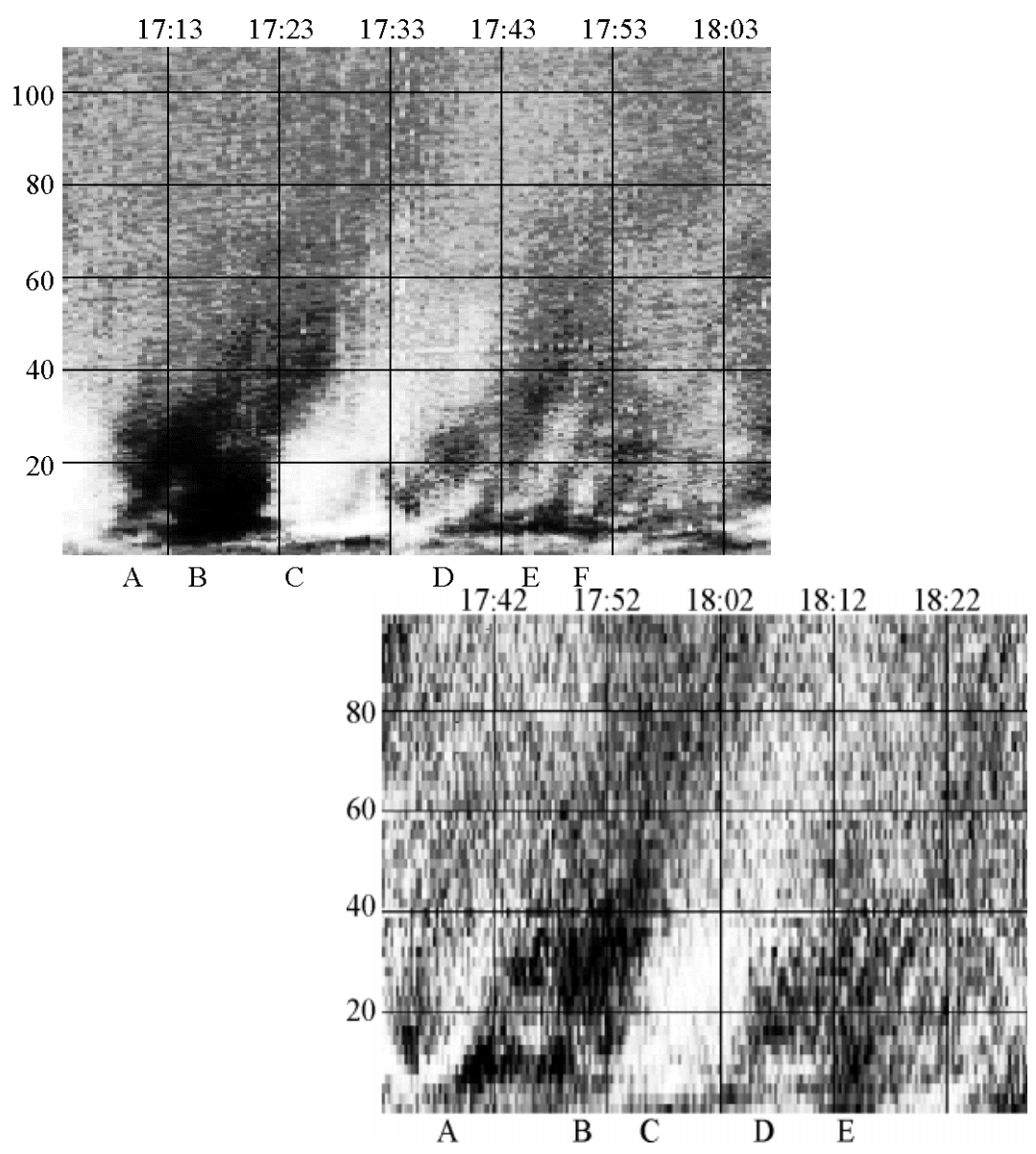

Fig. 5. The EUV intensity along path 1 as a function of time (horizontal axis) and distance along the path (vertical axis) in the TRACE data (upper) and in the EIT data (lower) tracked in Fig. 2

respect to the other PDs. PD Ti does not reach as high, therefore its total speed will be more dominated by lower height. In the TRACE frame starting at 17:28 at height $20 \mathrm{Mm}$ we can measure a period of $5 \mathrm{~min}$ between the PDs. In EIT we only seem to measure a period of $10 \mathrm{~min}$. There is a good overlap of measurements: Tf and Ea; Te and Eb; Ti and Ec. The expected projection angles match well. PDs Ec and Ed seem to be of a different population. Perhaps two loops are contained in the path. If we exclude Ec and Ed we find an average speed $120 \pm 4 \mathrm{~km} \mathrm{~s}^{-1}$ giving an angle of $\alpha_{\exp }=50 \pm 1^{\circ} \operatorname{good}$ corresponding to the average angle deduced from the TRACE data.

\subsubsection{Path 3}

The footpoint lies at $5 \mathrm{Mm}$ of the loop chosen. Clearly there are different speeds present in both diagrams. PDs $\mathrm{Ta}$ and $\mathrm{Tb}$ indicate an acceleration of propagation. In the EIT frame PDs Ef and Eg are quite uncertain, especially in the lower part of the diagram. There is an extreme good overlap between PD Te and Ed. They have the same structure and therefore probably have the same physical origin. Also the expected projection angles match well. $\mathrm{Pds}$ Ta and Ea both are broad and therefore difficult to measure. However, it seems possible that Ea is a periodical 
Path 2

\begin{tabular}{|c|c|c|c|c|c|c|c|c|c|}
\hline \multicolumn{5}{|c|}{ TRACE } & \multicolumn{5}{|c|}{ EIT } \\
\hline l & $v_{\mathrm{p}}(<20 \mathrm{Mm})$ & $v_{\mathrm{p}}(>20 \mathrm{Mm})$ & $v_{\mathrm{p}}$ & \multirow[t]{2}{*}{$\alpha_{\exp }$} & 1 & $v_{\mathrm{p}}(<20 \mathrm{Mm})$ & $v_{\mathrm{p}}(>20 \mathrm{Mm})$ & $v_{\mathrm{p}}$ & \multirow[t]{2}{*}{$\alpha_{\exp }$} \\
\hline & \multicolumn{3}{|c|}{$\left(\right.$ in $\left.\mathrm{km} \mathrm{s}^{-1}\right)$} & & & \multicolumn{3}{|c|}{$\left(\right.$ in $\left.\mathrm{km} \mathrm{s}^{-1}\right)$} & \\
\hline $\mathrm{A}$ & - & $64 \pm 4$ & $64 \pm 4$ & $63^{\circ}-67^{\circ}$ & - & - & - & - & - \\
\hline B & $124 \pm 19$ & $113 \pm 14$ & $107 \pm 4$ & $43^{\circ}-47^{\circ}$ & - & - & - & - & - \\
\hline $\mathrm{C}$ & $84 \pm 5$ & $105 \pm 7$ & $88.5 \pm 3$ & $53^{\circ}-56^{\circ}$ & - & - & - & - & - \\
\hline $\mathrm{F}$ & $73 \pm 6$ & $96 \pm 4$ & $93 \pm 15$ & $44^{\circ}-59^{\circ}$ & A & $62 \pm 7$ & $139 \pm 26$ & $112 \pm 6$ & $52^{\circ}-57^{\circ}$ \\
\hline $\mathrm{D}$ & $93 \pm 8$ & $111 \pm 7$ & $104 \pm 5$ & $44^{\circ}-50^{\circ}$ & - & - & - & - & - \\
\hline $\mathrm{E}$ & $100 \pm 13$ & $115 \pm 18$ & $102 \pm 4$ & $46^{\circ}-50^{\circ}$ & B & $111 \pm 14$ & $141 \pm 34$ & $127 \pm 5$ & $46^{\circ}-51^{\circ}$ \\
\hline G & $68 \pm 9$ & $97 \pm 8$ & $87 \pm 5$ & $53^{\circ}-57^{\circ}$ & - & - & - & - & - \\
\hline I & $74 \pm 3$ & - & $83 \pm 3$ & $58^{\circ}-56^{\circ}$ & $\mathrm{C}$ & $70 \pm 6$ & $123 \pm 13$ & $97 \pm 4$ & $58^{\circ}-61^{\circ}$ \\
\hline $\mathrm{H}$ & $81 \pm 13$ & $121 \pm 18$ & $83 \pm 6$ & $54^{\circ}-60^{\circ}$ & - & - & - & - & - \\
\hline- & - & - & - & - & $\mathrm{D}$ & $63 \pm 5$ & - & $80 \pm 14$ & $61^{\circ}-70^{\circ}$ \\
\hline $\mathrm{J}$ & $109 \pm 8$ & - & $117 \pm 9$ & $34^{\circ}-45^{\circ}$ & - & - & - & - & - \\
\hline- & - & - & - & - & $\mathrm{E}$ & $112 \pm 22$ & $127 \pm 21$ & $110 \pm 16$ & $49^{\circ}-61^{\circ}$ \\
\hline- & - & - & - & - & $\mathrm{F}$ & $100 \pm 17$ & $122 \pm 36$ & $107 \pm 14$ & $51^{\circ}-61^{\circ}$ \\
\hline - & - & - & - & - & G & $101 \pm 11$ & - & $105 \pm 13$ & $52^{\circ}-61^{\circ}$ \\
\hline
\end{tabular}

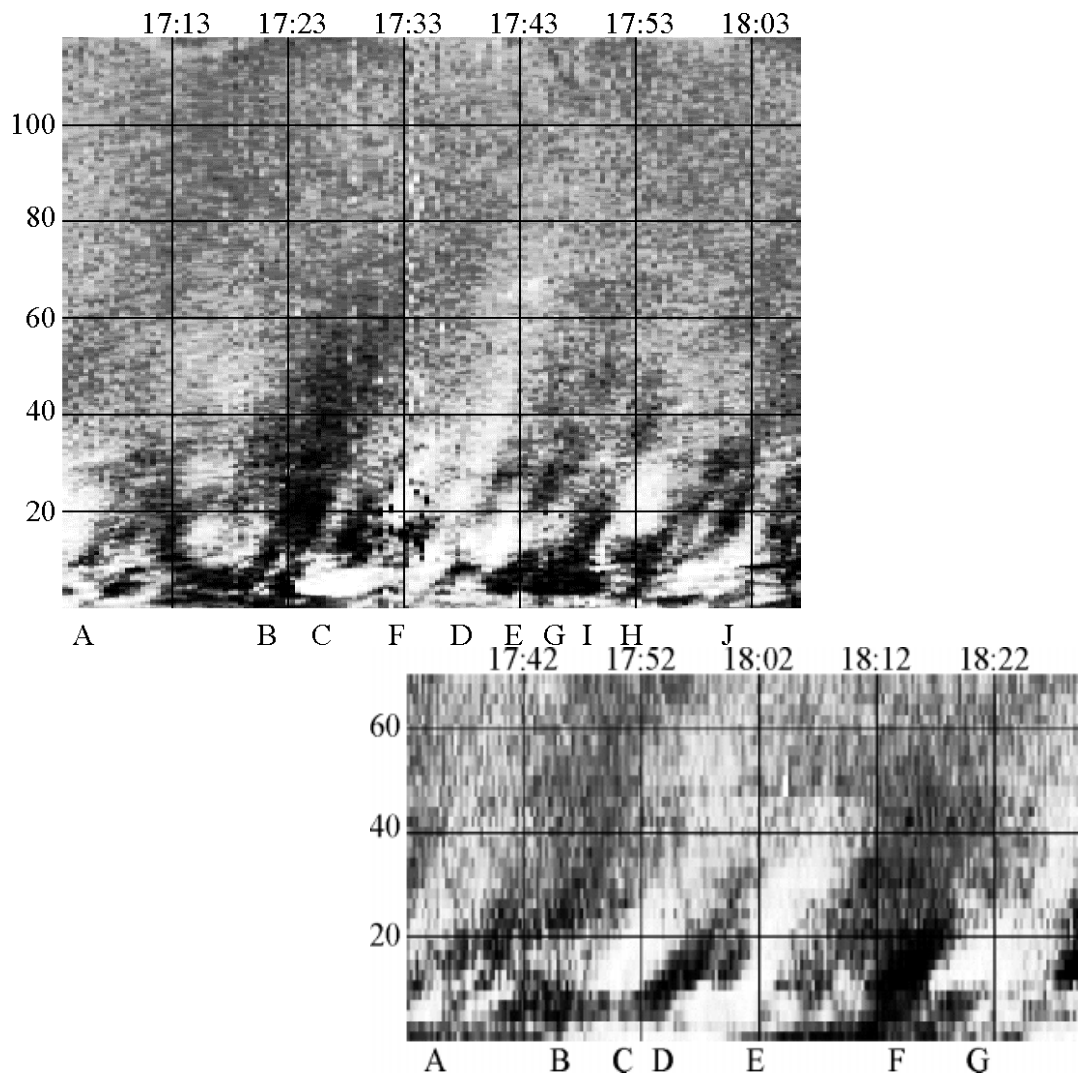

Fig. 6. The EUV intensity along path 2 as a function of time (horizontal axis) and distance along the path (vertical axis) in the TRACE data (upper) and in the EIT data (lower) tracked in Fig. 2

repetition of $\mathrm{PD} \mathrm{Ta}$. If this would be the case, we would measure a period of about $30 \mathrm{~min}$, which we also have found in Path 1. As mentioned in the table, there are two other pairs of matching PDs being Tc and $\mathrm{Eb}$, and $\mathrm{Td}$ and Ec.

\subsubsection{Path 4}

The footpoint is at about $10 \mathrm{Mm}$ of the starting point of the path. It is difficult to find similarities between the two diagrams. They seem to be different in nature. Only PDs Td and Ea seem to correspond. While we find clearly distinct disturbances propagating in the EIT diagram, the TRACE diagram seems to contain less PDs. In the TRACE frame there is no clear evidence for acceleration from overall view, but regarding the best $\mathrm{PD}, \mathrm{Ta}$, we do see acceleration. After the cosmic snow at $17 \mathrm{~h} 28$, speeds seem to be less than before. This is a trend seen with other paths as well. Maybe the nearby B flare shook the loop to a different position. 


\begin{tabular}{|c|c|c|c|c|c|c|c|c|c|}
\hline \multicolumn{5}{|c|}{ TRACE } & \multicolumn{5}{|c|}{ EIT } \\
\hline 1 & $v_{\mathrm{p}}(<20 \mathrm{Mm})$ & $v_{\mathrm{p}}(>20 \mathrm{Mm})$ & $v_{\mathrm{p}}$ & \multirow[t]{2}{*}{$\alpha_{\exp }$} & 1 & $v_{\mathrm{p}}(<20 \mathrm{Mm})$ & $v_{\mathrm{p}}(>20 \mathrm{Mm})$ & $v_{\mathrm{p}}$ & \multirow[t]{2}{*}{$\alpha_{\exp }$} \\
\hline & \multicolumn{3}{|c|}{$\left(\right.$ in $\left.\mathrm{km} \mathrm{s}^{-1}\right)$} & & & \multicolumn{3}{|c|}{$\left(\right.$ in $\left.\mathrm{km} \mathrm{s}^{-1}\right)$} & \\
\hline $\bar{A}$ & $54 \pm 5$ & $114 \pm 14$ & $87 \pm 12$ & $49^{\circ}-61^{\circ}$ & - & - & - & - & - \\
\hline B & $58 \pm 4$ & $111 \pm 10$ & $86 \pm 6$ & $52^{\circ}-58^{\circ}$ & - & - & - & - & - \\
\hline - & - & - & - & - & A & $124 \pm 11$ & $137 \pm 19$ & $124 \pm 13$ & $44^{\circ}-55^{\circ}$ \\
\hline $\mathrm{C}$ & $92 \pm 7$ & $105 \pm 5$ & $86 \pm 2$ & $54^{\circ}-57^{\circ}$ & B & $104 \pm 7$ & $106 \pm 12$ & $106 \pm 7$ & $54^{\circ}-59^{\circ}$ \\
\hline $\mathrm{D}$ & $89 \pm 7$ & $88 \pm 7$ & $86 \pm 8$ & $52^{\circ}-59^{\circ}$ & $\mathrm{C}$ & $111 \pm 12$ & $137 \pm 5$ & $112 \pm 3$ & $53^{\circ}-56^{\circ}$ \\
\hline $\mathrm{E}$ & $83 \pm 8$ & $107 \pm 13$ & $93 \pm 7$ & $48^{\circ}-56^{\circ}$ & $\mathrm{D}$ & $118 \pm 14$ & $139 \pm 27$ & $126 \pm 9$ & $45^{\circ}-53^{\circ}$ \\
\hline- & - & - & - & - & $\mathrm{E}$ & $129 \pm 24$ & $155 \pm 20$ & $124 \pm 12$ & $45^{\circ}-54^{\circ}$ \\
\hline - & - & - & - & - & $\mathrm{F}$ & $59 \pm 10$ & $112 \pm 4$ & $79 \pm 3$ & $64^{\circ}-67^{\circ}$ \\
\hline- & - & - & - & - & G & $46 \pm 4$ & $102 \pm 12$ & $70 \pm 10$ & $65^{\circ}-72^{\circ}$ \\
\hline- & - & - & - & - & $\mathrm{H}$ & $114 \pm 11$ & $131 \pm 10$ & $120 \pm 7$ & $48^{\circ}-54^{\circ}$ \\
\hline
\end{tabular}

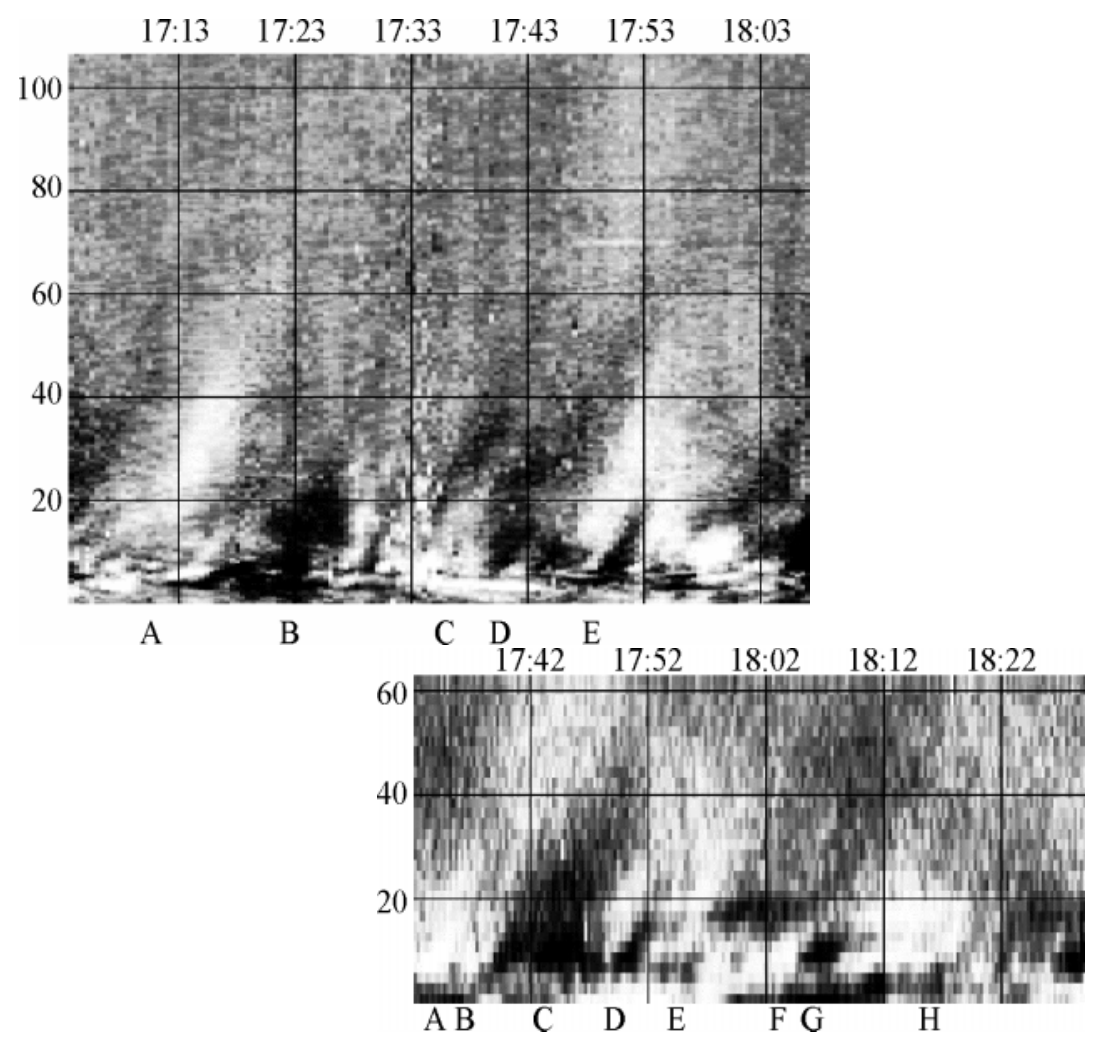

Fig. 7. The EUV intensity along path 3 as a function of time (horizontal axis) and distance along the path (vertical axis) in the TRACE data (upper) and in the EIT data (lower) tracked in Fig. 2

\subsection{Variability}

In order to quantify the variability in the loops, we define two different parameters for each pixel $(x, y)$ :

1. $\sigma_{\mathrm{a}}(x, y)$ : the standard deviation with respect to the median image, expressed in DN. This quantity is a measure of the combined effect of various temporal variability components, such as slow background trends, rapid transients and oscillatory fluctuations, but also random variations due to instrumental noise;

2. $\sigma_{\mathrm{n}}(x, y)$ : the expected instrumental noise level also expressed in DN. The main contributions to the instrumental noise are the photon shot noise (Poisson statistics), here calculated as a function of intensity $I$, and the $\mathrm{CCD}$ readout noise:

$$
\begin{aligned}
\sigma_{n} & =\sqrt{0.25 I+2.7}, & & \text { for } \operatorname{EIT}(195 \AA) ; \\
& =\sqrt{0.08 I+2.8}, & & \text { for TRACE }(171 \AA) ;
\end{aligned}
$$

with $I$ the observed intensity expressed in DN. At times of cosmic snow, the noise from cosmic-ray removal dominates. This occurs mainly in the TRACE data between 17:28 UT and 17:35 UT. For a more elaborate calculation of noise see Berghmans \& Clette (1999) for EIT and Aschwanden et al. (2000a) for TRACE.

The fractional variability $\sigma_{\mathrm{a}} / I$ is plotted in Fig. 9 for the 4 paths selected. We also plotted in dashed line the fractional instrumental noise $\sigma_{\mathrm{n}} / I$, and as dotted line three 
Path 4

\begin{tabular}{|c|c|c|c|c|c|c|c|c|c|}
\hline \multicolumn{5}{|c|}{ TRACE } & \multicolumn{5}{|c|}{ EIT } \\
\hline l & $v_{\mathrm{p}}(<20 \mathrm{Mm})$ & $v_{\mathrm{p}}(>20 \mathrm{Mm})$ & $v_{\mathrm{p}}$ & \multirow[t]{2}{*}{$\alpha_{\exp }$} & l & $v_{\mathrm{p}}(<20 \mathrm{Mm})$ & $v_{\mathrm{p}}(>20 \mathrm{Mm})$ & $v_{\mathrm{p}}$ & \multirow[t]{2}{*}{$\alpha_{\exp }$} \\
\hline & \multicolumn{3}{|c|}{$\left(\right.$ in $\left.\mathrm{km} \mathrm{s}^{-1}\right)$} & & & \multicolumn{3}{|c|}{$\left(\right.$ in $\left.\mathrm{km} \mathrm{s}^{-1}\right)$} & \\
\hline $\bar{A}$ & $78 \pm 10$ & $109 \pm 9$ & $93 \pm 4$ & $50^{\circ}-54^{\circ}$ & - & - & - & - & - \\
\hline B & $96 \pm 4$ & - & $100 \pm 7$ & $45^{\circ}-52^{\circ}$ & - & - & - & - & - \\
\hline $\mathrm{C}$ & $140 \pm 24$ & $100 \pm 9$ & $101 \pm 10$ & $43^{\circ}-53^{\circ}$ & - & - & - & - & - \\
\hline $\mathrm{F}$ & $109 \pm 9$ & $145 \pm 24$ & $122 \pm 8$ & $31^{\circ}-42^{\circ}$ & - & - & - & - & - \\
\hline - & - & - & - & - & A & $110 \pm 11$ & $165 \pm 34$ & $124 \pm 12$ & $45^{\circ}-54^{\circ}$ \\
\hline $\mathrm{D}$ & $66 \pm 3$ & $97 \pm 36$ & $86 \pm 3$ & $54^{\circ}-57^{\circ}$ & - & - & - & - & - \\
\hline- & - & - & - & - & B & $81 \pm 11$ & $101 \pm 5$ & $89 \pm 5$ & $60^{\circ}-64^{\circ}$ \\
\hline $\mathrm{E}$ & $79 \pm 3$ & $89 \pm 7$ & $80 \pm 3$ & $57^{\circ}-60^{\circ}$ & - & - & - & - & - \\
\hline- & - & - & - & - & $\mathrm{C}$ & $80 \pm 11$ & $105 \pm 7$ & $96 \pm 12$ & $56^{\circ}-64^{\circ}$ \\
\hline - & - & - & - & - & $\mathrm{D}$ & $99 \pm 11$ & $106 \pm 11$ & $102 \pm 10$ & $54^{\circ}-62^{\circ}$ \\
\hline - & - & - & - & - & $\mathrm{E}$ & $144 \pm 17$ & $131 \pm 14$ & $138 \pm 14$ & $38^{\circ}-50^{\circ}$ \\
\hline - & - & - & - & - & $\mathrm{F}$ & $99 \pm 28$ & $124 \pm 12$ & $98 \pm 17$ & $53^{\circ}-65^{\circ}$ \\
\hline
\end{tabular}

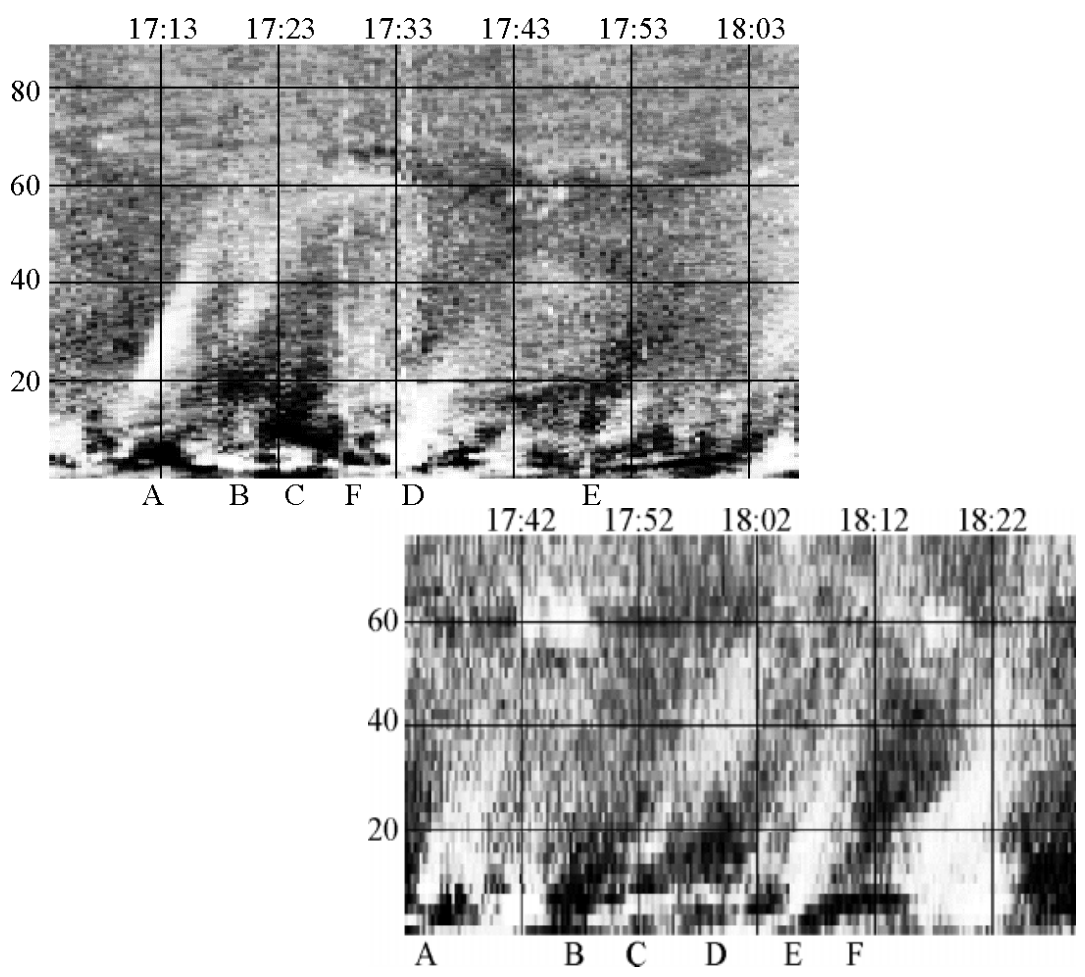

Fig. 8. The EUV intensity along path 4 as a function of time (horizontal axis) and distance along the path (vertical axis) in the TRACE data (upper) and in the EIT data (lower) tracked in Fig. 2

times the fractional instrumental noise. As mentioned previously, $\sigma_{\mathrm{a}}$ includes various variability components. Hence it cannot be interpreted as a measure of the change in amplitude due to the PD only. For example, the peak present in the plots corresponding to path 3 (EIT) illustrates the contribution from a brightening to the variability. In the category of the brightenings we also find possible indications of 5-min oscillations (Fig. 6). Other contributions to the variation of intensity are transverse loop oscillations. Their presence can be inferred from the difference images in Fig. 4. The places where field lines are sharply marked along the whole distance of the loop, indicate that the loops show a transverse motion.

\subsection{Characteristics of a typical Propagating Disturbance (PD)}

From different plots similar to Fig. 10, where the intensity during the whole sequence in a fixed point is plotted, we find the following characteristics of a PD:

1. The amplitude enhancements due to a passing disturbance are typically $8 \%$ and $12 \%$ for the $195 \AA$, resp. $171 \AA$, wavelength of the background intensity. By measuring the intensity in that point just before (or after) the PD passes we have an idea of the background intensity in a point;

2. Figure 10 can also be used to estimate the time a PD needs to cross a point, by measuring the width (in 

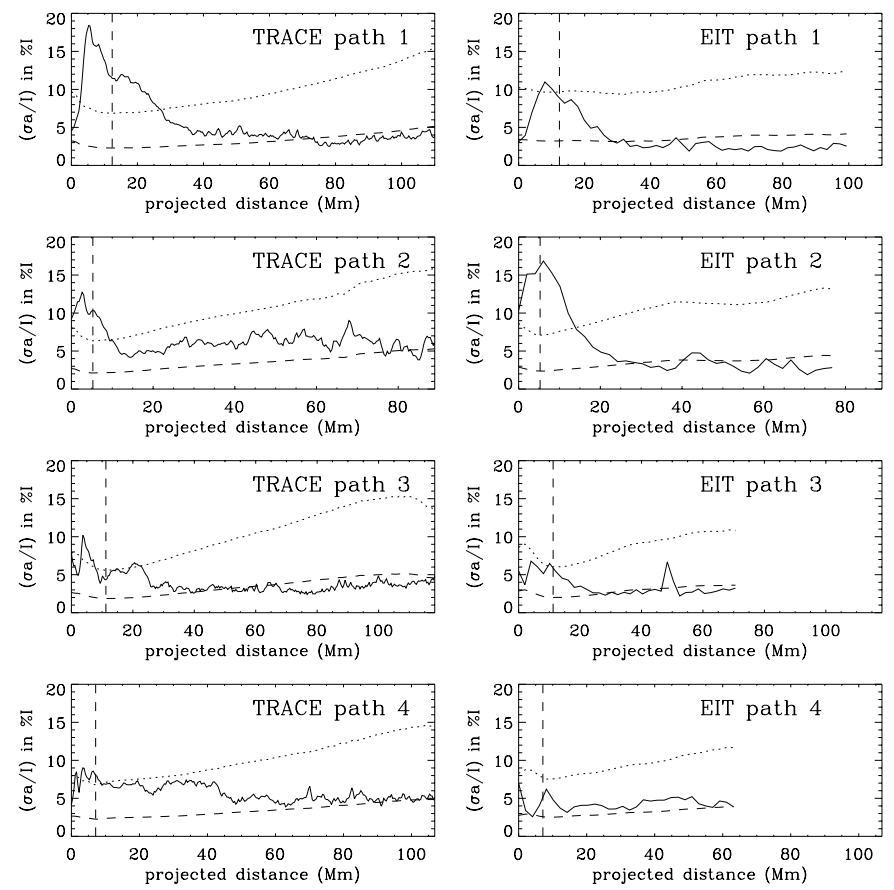

Fig. 9. The fractional variability $\left(\sigma_{\mathrm{a}} / I\right)$ for the selected loops expressed in $\% I$. The vertical line indicates the position of the footpoint. The dashed line corresponds to the instrumental noise $\sigma_{\mathrm{n}}$, the dotted line is three times the noise

time) of a disturbance. The typical duration of a PD passing a point in the TRACE data is $169 \mathrm{~s}$. This is not to be confused with the decay-time, which expresses the total life-time of a PD while traveling along the loop;

3. We do not exclude the possibility that the disturbances are triggered by pulses or even by a periodical signal at the footpoints. However it is hard to decide, because the PDs are not always clear;

4. The PDs differ from brightenings since they are not stationary. They travel along the loops with a roughly constant speed. Speeds are measured in a time-space diagram (see Figs. 5 to 8 ) by measuring the slope of the diagonal ridges. Typical velocities are $110 \mathrm{~km} \mathrm{~s}^{-1}$ for EIT and $95 \mathrm{~km} \mathrm{~s}^{-1}$ for TRACE. An acceleration of propagation is detected, in some cases, above the loop footpoint.

\section{Discussion}

We find outward propagating disturbances in virtually all loops within the opening bundle shown. Along some paths however we find more than one propagation speed, which we interpret as being due to the superposition of different loops.

In all cases studied, the PDs are weak disturbances, typically 8 and $12 \%$ of the background intensity resp. for the $195 \AA$ and $171 \AA$, bandpass, which corresponds to a change of $2.8 \%$ resp. $3.5 \%$ of the background density. This means they are marginally detectable above the noise
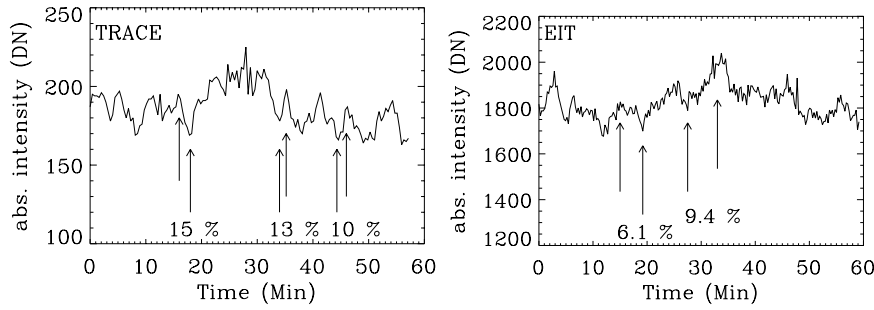

Fig. 10. The temporal evolution near the footpoint of path 4 . The absolute intensity is plotted. The arrows point towards the intensities used to calculate the amplitude enhancement w.r.t. the background intensity due to a passing disturbance

level. This typical amplitude is convolved with the varying throughput bandpass efficiency as a function of temperature. Although detailed temperature diagnostics are beyond the scope of the present paper, it seems most likely that these disturbances are formed closely to the peak formation temperature of the instruments bandpass. Suppose that a PD at a temperature significantly deviating from this peak formation temperature was detected, with an amplitude of the order of $10 \%$ of the background intensity. This would imply that another PD formed at the peak formation temperature, would have a significantly larger amplitude enhancement than $10 \%$ amplitude. None of this has been observed for many different PDs along many different loops neither in the present data set nor by other authors (e.g. De Moortel et al. 2000). So, at least as a working hypothesis, is seems reasonable to assume that the PDs are formed at the peak formation temperature of the bandpass through which they are observed (1.6 MK for $\operatorname{EIT}(195 \AA)$ and $1 \mathrm{MK}$ for TRACE $(171 \AA))$.

We can, in most cases, clearly distinguish a dominant direction of diagonal structure in all the time-space diagrams. This indicates that a dominant speed of propagation is present inside the loops. However, due to bad loop-tracking or perhaps the superposition of loops, we sometimes distinguish more than one velocity in an time-space diagram. Projected speeds found are typically $95 \mathrm{~km} \mathrm{~s}^{-1}$ for the $171 \AA$ line and $110 \mathrm{~km} \mathrm{~s}^{-1}$ for the $195 \AA$ line. The typical error on the measured speeds is $10 \mathrm{~km} \mathrm{~s}^{-1}$. The formal sound speed that correspond to these temperatures $\left(192 \mathrm{~km} \mathrm{~s}^{-1}\right.$ for EIT and $152 \mathrm{~km} \mathrm{~s}^{-1}$ for TRACE) are of the order of, but always higher than the observed propagation speeds. By interpreting the difference between the derived sound speed and the observed propagation speed as exclusively due to a projection effect, we can derive the necessary projection angle $\alpha_{\text {exp }}$. These expected projection angles are consistent with the observed angles when the bundle of loops crossed the western limb a few days later. This interpretation is possible since $\alpha_{\text {exp }}$ lies in the acceptable range deduced by Fig. 3 (right) and because of the good correspondence between the angles measured in both wavelengths.

In the loops investigated the speed measured at $195 \AA$ (EIT) is higher than the speed measured at $171 \AA$ (TRACE). Interpreting the PD as a slow mode wave traveling along the same loop simultaneously in the TRACE 
and EIT data set, we encounter here a conceptual problem: how can the same wave give signatures at different temperatures traveling at different speeds? This hints at sharp temperature gradients in the selected paths: either the loops consist of concentric shells at different temperatures (e.g. Foukal 1976) or each "observed loop" consists of a bundle of very thin loop threads each at a different temperature (e.g. Aschwanden et al. 2000b; Fludra et al. 1997; Matthews \& Harra-Murnion 1997). In both cases, the observations suggest that waves are triggered at the footpoints into the different shells or threads by the same source and propagate according to the sound speed of each shell or thread. Depending on the used bandpass this population of shells or threads is then convolved with the instrument bandpass, so that only those waves that travel along the loop at the peak formation temperature of the bandpass are seen. The observations show cases where the measured speeds of simultaneous PDs in both bandpasses are not consistent with the proposed explanation. This may be due to the superposition of loops where PDs from different depths in the field of view are compared, or due to less effective tracking of the loop due to transverse loop motions. Therefore, overlapping, multiwavelength observations of longer duration and improved data-analysis techniques are desirable.

Although our present data set does not allow to extend this reasoning much further, we are close here to coronal seismology: trying to determine the internal coronal loop structure by studying the characteristics of the waves passing through it.

An alternative interpretation of the PDs as compared to the slow mode view is the hypothesis of plasma flows along the loop. Aschwanden et al. (2000) concluded from the properties of extensive loop systems as observed by TRACE, that they cannot be in static equilibrium, but instead harbour continual chromospheric upflows. The flow accelerates with height and may even become supersonic. The observation we have presented here show no systematic acceleration of disturbances along the loop. The propagation speed of the PDs remain reasonably constant. Similar discussions on these two broad categories of interpretation (wave model vs. flow model) exist (e.g. Brekke et al. 1997).

As found in previous studies (e.g. Svestka et al. 1982a, $1982 \mathrm{~b}$ ) we find evidence of quasi-periodical triggering of the PDs at the footpoints. Reconnection caused by a flare as was found in e.g. Aschwanden et al. (1999) and Nakariakov et al. (1999) is also a valuable candidate as excitation mechanism. In a first inventory of this active region, based on the EIT data, there are only one strong brightening and several small brightenings detected around the footpoints of the loops here discussed (see Berghmans \& Clette 1999).

We aim to compare the observations to analytical models which model the propagation of sound waves in a coronal, isothermal loop structure, and study the feasibility of coronal diagnostics by such a method of comparison (Nakariakov et al. 2000).
Acknowledgements. The authors would like to thank the whole JOP80 team for their contribution to this unique data set. E. Verwichte, D. Berghmans and J. F. Hochedez would like to acknowledge the support of the Belgian Federal Services of Scientific, Technical and Cultural Affairs (SSTC/DWTC).

\section{References}

Aschwanden, M. J., Fletcher, L., Schrijver, C. J., \& Alexander, D. 1999, ApJ, 520, 880

Aschwanden, M. J., Nightingale, R. W., Tarbell, T. D., \& Wolfson, C. J. 2000, ApJ, 535, 1027

Aschwanden, M. J., Nightingale, R. W., \& Alexander, D. 2000, ApJ, 541, 1059

Berghmans, D., Clette, F., \& Moses, D. 1998, A\&A, 336, 1039

Berghmans, D., \& Clette, F. 1999, Sol. Phys., 186, 207

Brekke, P., Kjeldseth-Moe, O., \& Harrison, R. A. 1997, Sol. Phys., 175, 511

Clette, F., et al. 1998, http://sohowww . nascom. nasa.gov/soc/JOPs/jop080.txt

Delaboudinière, J.-P., et al. 1995, Sol. Phys., 162, 291

De Moortel, I., Ireland, J., \& Walsh, R. W. 2000, A\&A, 355, L23

DeForest, C. E., \& Gurman, J. B. 1998, ApJ, 501, L217

Fludra, A., et al. 1997, Sol. Phys., 175, 487

Foukal, P. V. 1976, ApJ, 210, 575

Handy, B. N., et al. 1999, Sol. Phys., 187, 229

Harrison, R. A. 1987, A\&A, 182, 337

Harrison, R. A. 1997, in Proc of the 5th SoHO Workshop, Oslo, June 1997, ESA SP-404, 7

Harrison, R. A. 1997, Sol. Phys., 175, 467

Innes, D. E., Inhester, B., Axford, W. I., \& Whilhelm, K. 1997, Nature, 386(24), 811

Kjeldseth-Moe, \& Brekke, P. 1998, Sol. Phys., 182, 73

Martin, S. F., \& Svestka, Z. F. 1988, Sol. Phys., 116, 91

Matthews, S. A., \& Harra-Murnion, L. K. 1997, Sol. Phys., 175,541

Nakariakov, V. M., Ofman, L., Deluca, E., Roberts, B., \& Davila, J. M. 1999, Science, 285, 862

Nakariakov, V. M., Verwichte, E., Berghmans, D., \& Robbrecht, E. 2000, A\&A, 362, 1151

Nightingale, R. W., Aschwanden, M. J., \& Hurlburt, N. E. 1999, Sol. Phys., 190, 249

Ofman, L., Nakariakov, V. M., \& DeForest, C. E. 1999, ApJ, 514,441

Ofman, L., Nakariakov, V. M., \& Sehgal, N. 2000, ApJ, 533, 1071

Priest, E. 1984, Solar Magnetohydrodynamics (D. Reidel Publ. Co., Dordrecht, Holland)

Reale, F., Peres, G., \& Serio, S. 2000, ApJ, 535, 412

Robbrecht, E., Berghmans, D., \& Poedts, S. 1999, Proc. of the 8th SOHO Workshop, Paris, June 1999, ESA SP-446, 575

Roberts, B., Edwin, P. M., \& Benz, A. O. 1984, ApJ, 279, 857

Shimojo, M., Shibata, K., Hori, K., \& Yokoyama, T. 1998, Proc. of an international conference meeting in Guadeloupe, France, ESA SP-421, 163

Svestka, Z., et al., and 8 co-authors, 1982, Sol. Phys., 75, 305

Svestka, Z., et al. 1982, Sol. Phys., 80, 143

Svestka, Z., Farnik, F., Fontenla, J. M., \& Martin, S. F. 1989, Sol. Phys., 123, 317

Svestka, Z. 1994, Sol. Phys., 152, L505

Tarbell, T. 1994, Progress Report, Lockheed Missiles and Space Co. 1994 